\title{
S100A1 is Involved in Myocardial Injury Induced by Exhaustive Exercise
}

\author{
Authors \\ Miaomiao Yang ${ }^{1,2^{*}}$, Zhigang Xiao ${ }^{1,3^{*}}$, Zhaoli Chen ${ }^{1 *}$, Yongxin $\mathrm{Ru}^{4}$, Jun Wang ${ }^{5}$, Jianhua Jiang ${ }^{1}$, Xinxing Wang1(D, \\ Tianhui Wang1, 2
}

\section{Affiliations \\ 1 Tianjin Institute of Environmental and Operational Medicine, Tianjin 300050, China \\ 2 Tianjin Key Lab of Exercise Physiology and Sports Medicine, Tianjin University of Sport, Tianjin 301617, China \\ 3 School of Materials Science and Engineering, Tianjin University of Technology, Tianjin 300384, China \\ 4 Institute of Hematology and Blood Diseases Hospital Peaking Union Medical College, Tianjin 300020, China \\ 5 Air Force Medical Center, Medical Evaluation Depart- ment, Beijing 100042, China}

Key words

oxidative stress, exhaustive exercise, mitochondrion

accepted $\quad 08.09 .2021$

published online $\quad 23.10 .2021$

\section{Bibliography}

Int J Sports Med 2022; 43: 444-454

DOI $10.1055 / \mathrm{a}-1642-8352$

ISSN $0172-4622$

(C) 2021. Thieme. All rights reserved. Georg Thieme Verlag, Rüdigerstraße 14, 70469 Stuttgart, Germany

\section{Correspondence}

Dr. Tianhui Wang

Tianjin Institute of Environmental and Operational Medicine

1 Dali Road

Heping District

300050 Tianjin

P. R. China

Tel.: + 86/22/84655 322 , Fax: + 86/22/84655 018

wydny668@163.com
Dr. Xinxing Wang

Tianjin Institute of Environmental and Operational Medicine

1 Dali Road

Heping District

300050 Tianjin

P. R. China

Tel.: + 86-22-84655206, Fax: + 86-22-8465501

wxxemail@sina.cn

\section{ABSTRACT}

Many studies have confirmed that exhaustive exercise has adverse effects on the heart by generating reactive oxygen species (ROS). S100A1 calcium-binding protein A1 (S100A1) is a regulator of myocardial contractility and a protector against myocardial injury. However, few studies have investigated the role of S100A1 in the regulation of myocardial injury induced by exhaustive exercise. In the present study, we suggested that exhaustive exercise led to increased ROS, downregulation of S100a1, and myocardial injury. Downregulation of S100a1 promoted exhaustive exercise-induced myocardial injury and overexpression of S100A1 reversed oxidative stress-induced cardiomyocyte injury, indicating $\mathrm{S} 100 \mathrm{~A} 1$ is a protective factor against myocardial injury caused by exhaustive exercise. We also found that downregulation of S100A1 promoted damage to critical proteins of the mitochondria by inhibiting the expression of Ant1, Pgc1a, and Tfam under exhaustive exercise. Our study indicated $\mathrm{S} 100 \mathrm{~A} 1$ as a potential prognostic biomarker or therapeutic target to improve the myocardial damage induced by exhaustive exercise and provided new insights into the molecular mechanisms underlying the myocardial injury effect of exhaustive exercise.

These authors contributed equally to this work. 


\section{LIST OF ABBREVIATIONS}

$\begin{array}{ll}\text { EE } & \text { Exhaustive Exercise } \\ \text { SOD } & \text { Superoxide Dismutase } \\ \text { GSH-PX } & \text { Glutathione peroxidase } \\ \text { IHC } & \text { Immunohistochemistry } \\ \text { ROS } & \text { Reactive Oxygen Species } \\ \text { CK } & \text { Creatine Kinase } \\ \text { SiRNAs } & \text { Small Interfering RNAs } \\ \text { OCR } & \text { Oxygen Consumption Rate (OCR) } \\ \text { PGC-1 } \alpha & \text { Peroxisome proliferator-activated receptor } \\ & \text { gamma coactivator 1-alpha } \\ \text { ANT } & \text { Adenine Nucleotide Translocase } \\ \text { S100A1 } & \text { S100 calcium-binding protein A1 } \\ \text { TFAM } & \text { Transcription Factor A, Mitochondrial }\end{array}$

\section{Introduction}

The heart consumes the most energy in the cardiovascular system, allowing it to provide the necessary oxygen for various tissues and organs to maintain the normal metabolic and functional activities of the human body [1]. The benefits of physical exercise to the cardiovascular system have been reported [2,3]. However, unrestricted increase in the time and intensity of exercise might not always bring about more benefits to the cardiovascular system [4]. The heart is also one of the most sensitive organs to overtraining [1]. Many studies have confirmed that exhaustive exercise (EE) has adverse effects on the heart [5-7]. Exhaustive exercise not only causes a reduction in cardiac function and electro cardio-electric changes, but also leads to destruction of the myocardial ultrastructure and abnormal energy metabolism [1]. Exercise-induced myocardial injury has become a current focus of sports medicine.

Reactive oxygen species (ROS) and overproduction of free radicals are considered to be the most important causes of multiple tissue injury during EE $[1,8]$. Mitochondria, as the key cell organelles responsible for energy production and the control of many processes from signaling to cell death, are also important sites of ROS and free radical production [9]. Mitochondria are also important target organelles of oxidative stress. The heart has some of the highest mitochondrial densities of tissues found within the body [10]. As such, the higher oxidative capacity of the heart suggests it has higher potential to oxidative stress. Oxidative stress and mitochondrial dysfunction have been extensively studied and are considered targets of various pathophysiological processes [11, 12]. One of the explanations for ROS production by mitochondria is an excessive increase in energy demand. The mitochondrial respiratory chain constitutes the main intracellular source of ROS in most tissues [13]. It has been reported that complex I and complex III of the respiratory chain are responsible for ROS production, and also a number of other mitochondrial oxidoreductases producing hydrogen peroxide and/or superoxide radical [14]. Thus, studying the relationship between mitochondrial function and oxidative stress during $E E$ is beneficial to deepen our understanding of the mechanism of myocardial injury induced by EE.
S100A1, also known as S100 calcium-binding protein A1, is highly expressed in cardiac and skeletal muscle, and localizes to Z-discs and the sarcoplasmic reticulum. Early studies showed that S100A1 overexpression enhances cardiac contractile performance and established the concept of S100A1 as a regulator of myocardial contractility [15]. S100A1 released from ischemic cardiomyocytes can signal myocardial damage via Toll-like receptor 4 [16]. S100A 1 has been used as a target for gene therapy in the rodent model following acute myocardial infarction [17]. These studies suggested that S100A1 plays protective role when cardiomyocytes are damaged. However, studies on EE, myocardial injury, S100A1 and oxidative stress are limited.

Therefore, the aims of the present study were to explore: (1) the effect of EE on myocardial injury, S100A1, and oxidative stress; (2) the role of the $\mathrm{S100A} 1$ in oxidative stress-induced cardiomyocyte injury; and (3) the mechanism by which S100A1 regulates mitochondrial function and oxidative stress. Our results will increase our understanding of the precise role of S100A1 in cardiomyocyte injury induced by EE and will promote the potential clinical applications of this protein as a diagnostic or prognostic biomarker.

\section{Materials and Methods}

\section{Animals and treatments}

This study was conducted with the approval of the Animal Care and Use Committee of the Tianjin University of Sport, China. Animal care was performed in accordance with the China Laboratory Animal Management Regulations, the Guide for the Care and Use of Laboratory Animals (Institute for Laboratory Animal Research, Washington, DC, USA), and the Ethical Standards in Sport and Exercise Science Research: 2020 Update [18]. The protocol was approved by the Committee on the Ethics of Animal Experiments of Tianjin University of Sport. All surgery was performed under sodium pentobarbital ( $1 \%, 1 \mathrm{ml} / 100 \mathrm{~g}$ weight, i.p.) anesthesia, and all efforts were made to minimize animal suffering.

Male Wistar rats (56 days old, about $220 \mathrm{~g}$ ) were used for the experiment, and twelve rats were randomly divided into two groups (6 rats/group): control and exhaustive exercise (EE). Rats were acclimatized to their surroundings for 1 week before the start of the experiment. The animals were maintained on a $12 \mathrm{~h}$ light/ dark cycle under a controlled temperature of $25 \pm 2{ }^{\circ} \mathrm{C}$. Food and water were available for the duration of the experiments unless otherwise noted. All animal handling procedures were performed in strict accordance with the guide for the use and care of laboratory animals. Exhaustive exercise was performed as described in previous publications [19-21]. Briefly, two groups followed by 10 days of adaptive exercise training on the small animal platform. And on the 11th day, rats in EE group were trained for exhaustion exercise. First, rats were trained with a slope of $0^{\circ}$ and a speed of $9 \mathrm{~m} / \mathrm{min}$ for 15 minutes. Then, the rats were trained with a slope of $5^{\circ}$ and a speed of $15 \mathrm{~m} / \mathrm{min}$ for 15 minutes. Finally, the slope of the rat training was adjusted to $10^{\circ}$ and the speed was adjusted to $20 \mathrm{~m} / \mathrm{min}$, until they were exhausted. When the rat could not run further under the condition of electrical stimulation, it was determined to be exhausted. After exhausted exercise, all rats were humanely euthanized with sodium pentobarbital [22]. The plasma 
samples and myocardial tissues were collected for western blotting, immunohistochemistry, and enzyme linked immunosorbent assay (ELISA).

\section{Cell culture and treatments}

$\mathrm{H} 9 \mathrm{c} 2$ cells (rat embryonic cardiomyoblast-derived H9c2 cardiomyocytes) were maintained at $37^{\circ} \mathrm{C}$ in a $5 \% \mathrm{CO}_{2}$ incubator with Dulbecco's modified Eagle's medium (DMEM; Sigma-Aldrich Corporation, St. Louis, MO, USA; D6429) supplemented with $10 \%$ fetal bovine serum (FBS; Atlanta Biologicals, Flowery Branch, GA, USA; S12450), 2 mmol L-glutamine (GIBCO, Grand Island, NY, USA; 25030-081), and $1 \%$ penicillin/streptomycin (Life Technologies, Carlsbad, CA, USA; 15140163).

Oxidative stress is one of the most important ways of cardiomyocytes injury induced by exhaustive exercise [23]. The oxidative stress model induced by hydrogen peroxide $\left(\mathrm{H}_{2} \mathrm{O}_{2}\right)$ is often used to study the myocardial injury induced by exhaustive exercise [24]. For oxidative stress, $\mathrm{H} 9 \mathrm{c} 2$ cells were exposure to $\mathrm{H}_{2} \mathrm{O}_{2}(1 \mathrm{mM})$ for $2,4,6,12,24$ hours at approximately $80 \%-90 \%$ confluency $[25,26]$.

\section{Constructs and reagents}

$\mathrm{H} 9 \mathrm{c} 2$ cells were obtained from the Cell Bank of the Chinese Academy of Sciences (Shanghai, China). The S100A1 overexpression plasmid was purchased from Open Biosystems, Inc. (Lafayette, CO, USA). Lipofectamine 2000 was purchased from Life Technologies (Grand Island, NY, USA). The TRIzol reagent (15596026) and FirstStrand Synthesis system (18080051) was bought from Invitrogen Corporation (Waltham, MA, USA). Sequences of the S100a1 siRNA oligonucleotides were 5'-CUU CUG UCA AGA ACC UGC UTT-3' and 5'-AGC AGG UUC UUG ACA GAA GTT-3'. The antibodies specific against S100A1 (5066 s) were purchased from Cell Signaling Technology (Beverly, MA, USA). The antibodies specific against ANT1 (ab102032), PGC-1 $\alpha$ (ab54481), and Tfam (ab131607) were obtained from Abcam (Cambridge, MA, USA).

\section{Quantitative real-time PCR ( $q P C R$ )}

Total RNA was extracted from cells using the TRIzol reagent. Total cDNAs were synthesized using the RT-PCR system (Invitrogen Corporation; 11146-057). Real-time PCR was conducted following the protocol for the Fast SYBR Green Master Mix kit (Applied Biosystems, Foster City, CA, USA; 4385614) in a 7900HT Fast Real-Time PCR System (Applied Biosystems). The primers for Pgc1a (encoding PPARG coactivator 1 alpha) were $5^{\prime}$-TGG AGT GAC ATA GAG TGT GCTG-3' and 5'-TAT GTT CGC GGG CTC ATT GT-3'; for Tfam (encoding transcription factor $A$, mitochondrial) were $5^{\prime}$-TCA TGA CGA GTT CTG CCG TT-3' and 5'-CTT CAC AAA CCC GCA CGA AA-3'; for S100a 1 were $5^{\prime}$-AAA GAC CTG CTA CAA ACT GA-3' and 5'-CAC CAG CAC AAC AAA CTC C-3'; for Ant (encoding adenine nucleotide translocase) were 5'-CGC TAC TTC GCT GGT AAC CT-3' and 5'-ATG ATG CCC TGC ACA GAG AC-3'; and for Gapdh (encoding glyceraldehyde-3-phosphate dehydrogenase) were 5'-CCC CCA ATC AAT CCG TTG TG-3' and 5'-TAG CCC AGG ATG CCC TTT AGT-3'. Quantitative analysis was conducted as previously reported [27].

\section{Fluorescence imaging of ROS generation}

Thirty minutes before imaging, cells were fed with phenol red free DMEM, loaded with Dichloro-dihydro-fluorescein diacetate (DCFHDA) $\left(10 \mu \mathrm{M}\right.$ : Sigma Aldrich) in the dark, and kept in a $\mathrm{CO}_{2}$ incubator at $37^{\circ} \mathrm{C}$. Cells were then washed with DMEM and examined under a fluorescence microscope (Thermo Fisher Technology Co. LTD, Waltham, MA, USA) [28-30].

\section{MDA, SOD, GSH-PX, and CK content analysis}

The contents of malondialdehyde (MDA), superoxide dismutase (SOD), glutathione peroxidase (GSH-PX), and creatine kinase (CK) in the cell cultures were measured using enzyme-linked immunosorbent assay (ELISA) kits (A003-1, A001-2, A005 and A032, Nanjing Jiancheng Bioengineering Institute, Nanjing, China). ELISA was performed according to the manufacturer's instructions: The cell culture was added into a 96 -well plate $(100 \mu \mathrm{L} /$ well $)$, which was then sealed with parafilm and incubated at $37^{\circ} \mathrm{C}$ for $90 \mathrm{~min}$. The antibody $(100 \mu \mathrm{L} /$ well $)$ was added and incubated for $60 \mathrm{~min}$. The enzyme-binding solution ( $100 \mu \mathrm{L} /$ well) was added and incubated for a further $30 \mathrm{~min}$. The plate was washed four times and the optical absorption was estimated at $450 \mathrm{~nm}$ using a microplate reader (Bio-Rad, Hercules, CA, USA).

\section{Hematoxylin-eosin staining (HE) and immunohistochemistry (IHC)}

The left ventricle was used to hematoxylin-eosin staining as previously described [31]. Antibody specific against S100A1 (Cell Signaling, Cat NO: $5066 \mathrm{~s}$ ) was used for IHC, and the protein expression levels of the left ventricle were analyzed as previously described [32].

\section{Electron microscopy analysis}

Electron microscopy analysis was conducted as previously described [33]. Briefly, the myocardial tissue less than 1 cubic millimeter was fixed in $2.5 \%$ glutaraldehyde phosphate buffer for 2 hours. Then, wash with $0.1 \mathrm{M}$ phosphoric acid rinse solution three times (15 minutes / time), fix with $1 \%$ osmic acid fixed solution for $2-3$ hours, and wash with $0.1 \mathrm{M}$ phosphoric acid rinse solution (15 minutes / time). Next, ethanol is dehydrated, acetone is embedded and dried. The slices were cut into $70 \mathrm{~nm}$ thick sections and stained with $3 \%$ uranyl acetate and lead citrate. Finally, the images were observed and photographed by transmission electron microscope JEOL JEM-1230 (80 kV).

\section{Seahorse methods}

Oxygen consumption rate (OCR) and extracellular acidification rate (ECAR) were measured as previously described [34] using a Seahorse XFe24 (Agilent Technologies). Before testing, DMEM medium without glucose, pyruvate, and glutamax was used to as cell culture medium, and the cells was incubated at $37^{\circ} \mathrm{C}$ for $1 \mathrm{~h}$ without $\mathrm{CO}_{2}$ in a dry incubator. For OCR and ECAR measurements, oligomycin is used to block ATP synthesis and measure proton leakage, FCCP is used to uncouple respiration, and antimycin $A$ is used to suppress electron transfer. First, three basal measurements of OCR and ECAR were recorded, then $1 \mathrm{mM}$ oligocmycin, $1 \mathrm{mM} \mathrm{FCCP,}$ and $1 \mathrm{mM}$ antimycin A were injected successively. During this period, proton leak, maximal respiration, and non-mitochondrial respiration were recorded in turn. Reserve capacity is the difference 

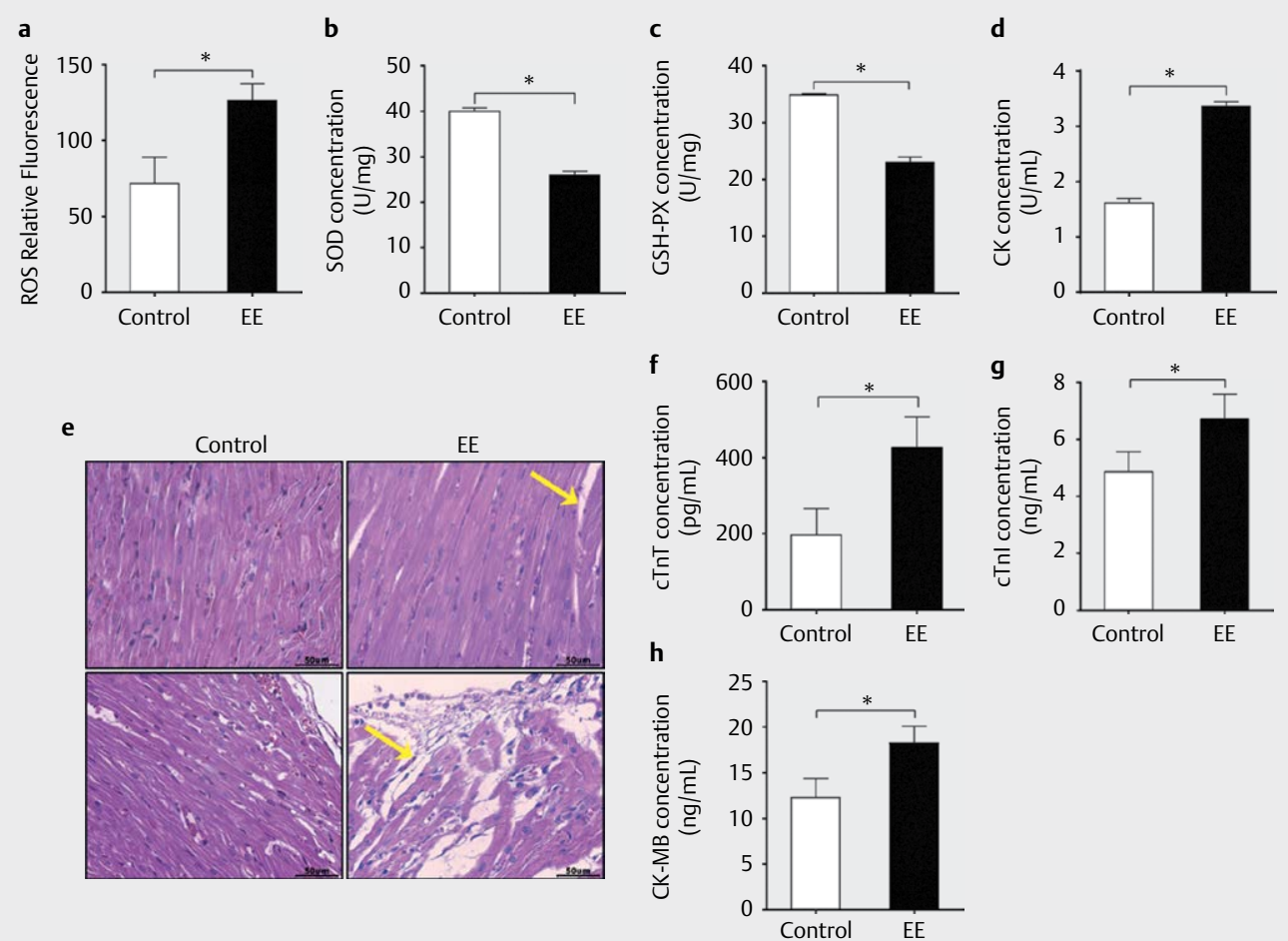

- Fig. 1 Exhaustive exercise leads to oxidative stress and myocardial injury in rats. (a) ROS levels were detected in the plasma of rats in the control and EE groups using fluorescence. (b-c) The plasma of rats in the control and EE groups was subjected to ELISA to determine the SOD and GSH-PX concentrations. (d) The CK concentration was detected in the plasma of rats in control and EE groups using ELISA. (e) The myocardial tissues in rats in the control and EE groups were detected by HE staining. (f) Concentration of cTnT in rats' serum; (g) Concentration of cTnl in rat's serum; (h) Concentration of CK-MB in rats' serum. Wilcoxon rank sum test was used to determine the significance of differences between two groups $(n=5)$. An asterisk $\left({ }^{*}\right)$ indicates a significant change compared with that in the control group $(P<0.05)$. Each experiment was repeated six times.

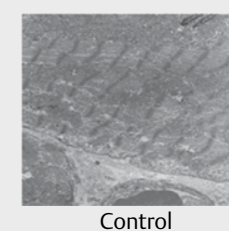

c

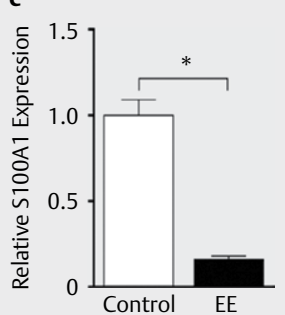

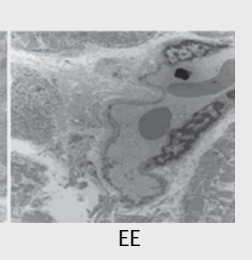

d

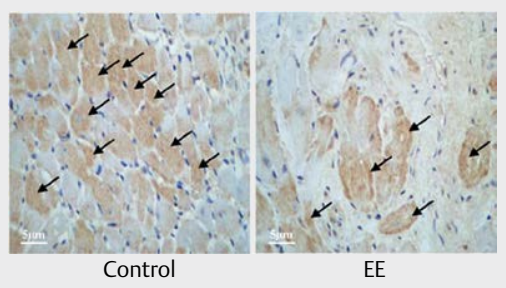

b
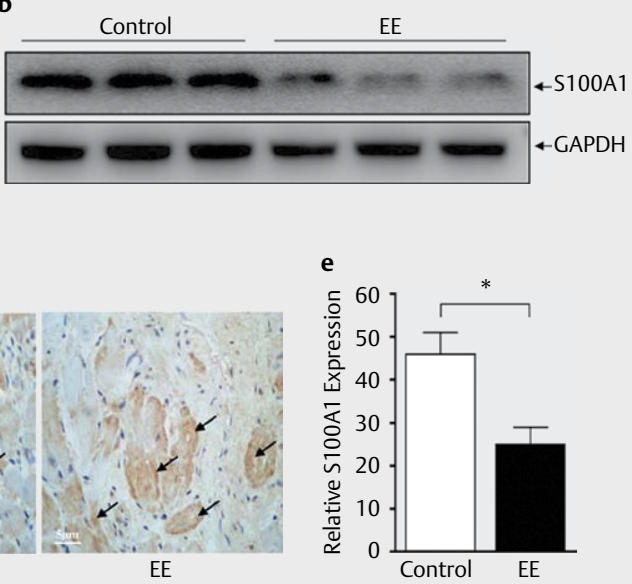

- Fig. 2 Exhaustive exercise promotes myocardial mitochondria injury and downregulation of S100A1 in rats. (a) The myocardial tissues of rats in the control and EE groups were examined using electron microscopy. (b) The myocardial tissue extracts were subjected to western blotting to determine S100A1 levels. GAPDH was used as a protein loading control. (c) Quantitative analysis of the S100A1 level in (B) by Image J. An asterisk ( ${ }^{*}$ ) indicates a significant change compared with that in the control group $(P<0.05, n=3)$. (d) $S 100 A 1$ levels detected using immunohistochemistry. (e) Quantitative analysis of the S100A1 level in (D) by Image J (https://imagej.en.softonic.com/). Calculate the integral optical density of the brown area in the picture and the area of the target distribution area to get the average optical density value, which is used to represent the S100A1 level of the sample. 
a

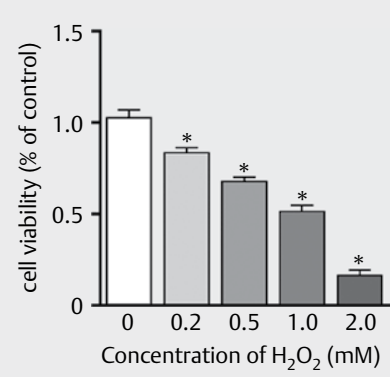

d

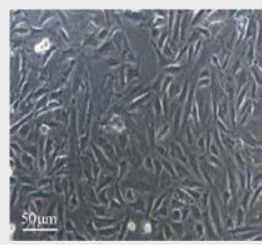

Control

e

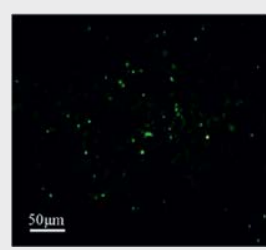

Control

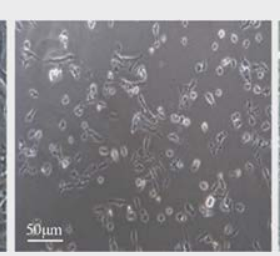

$\mathrm{H}_{2} \mathrm{O}_{2}$

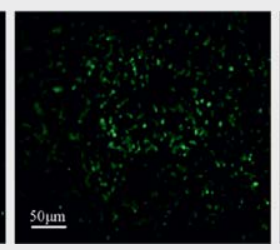

$\mathrm{H}_{2} \mathrm{O}_{2}$
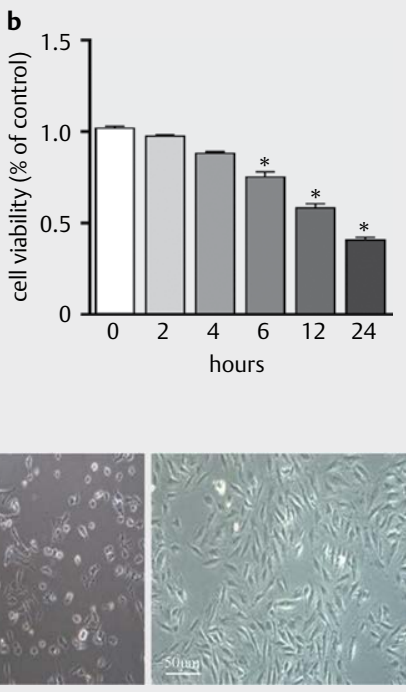

$\mathrm{H}_{2} \mathrm{O}_{2}+\mathrm{S} 100 \mathrm{~A} 1$

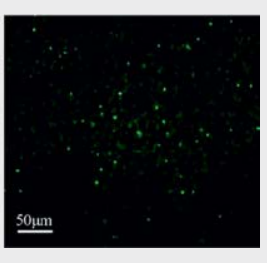

$\mathrm{H}_{2} \mathrm{O}_{2}+\mathrm{S} 100 \mathrm{~A} 1$

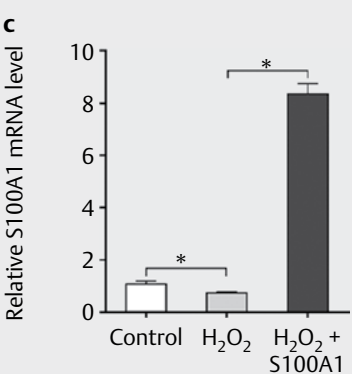

f

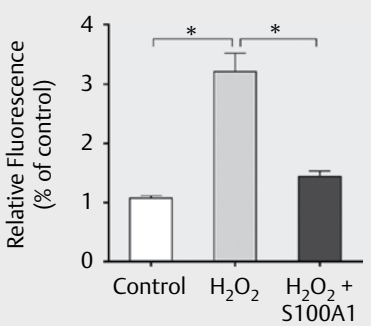

g
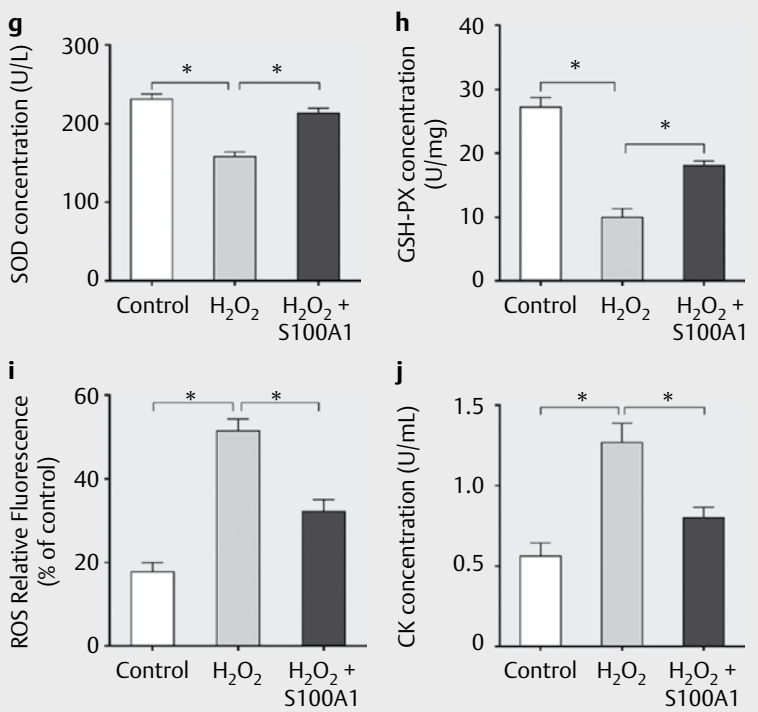

between maximal respiration and basal respiration, while ATPlinked OCR is the difference between basal and proton leak.

\section{Western blotting}

Antibodies specific for S100A1, ANT1, PGC-1 $\alpha$, and Tfam were obtained from Abcam (Cambridge, MA, USA). Anti-tubulin and Anti-GAPDH antibodies were purchased from Bioworld Technology, Inc. (Bloomington, MN, USA). Western blotting was performed as described in a previous publication [35]. Specifically, tissues or cells were lysed in RIPA buffer (Solaibao Biotechnology Co., Ltd., Beijing, China) supplemented with phenylmethylsulphonylfluoride (PMSF, Solaibao Biotechnology Co., Ltd., Beijing, China) to obtain total protein. Total protein concentrations were determined by using BCA Assay Kit (Solaibao Biotechnology Co., Ltd., Beijing, China). Samples in an equal volume of $5 X$ sample loading buffer were boiled $\left(100^{\circ} \mathrm{C}, 10 \mathrm{~min}\right)$ in loading buffer. Samples were loaded on the polyacrylamide gel (15\%) along with the standard marker proteins and the electrophoresis was run with supply of $250 \mathrm{~mA}$ current (100 min), followed by transfer to a PVDF membrane (Millipore, USA). After being blocked with $5 \%$ nonfat dry milk for $2 \mathrm{~h}$ at room temperature, the membranes were incubated with 1:1000-diluted primary antibody overnight at $4{ }^{\circ} \mathrm{C}$. After washed $10 \mathrm{~min}$ in triplicate with the TBS-T buffer, the membranes were incubated with goat anti-rabbit secondary antibody at room temperature for $1 \mathrm{~h}$. Membranes were washed three times for $10 \mathrm{~min}$ and treated with enhanced chemiluminescence (ECL) reagent. Autoexposure settings were used to get protein bands. Meanwhile, the optical intensity of the bands was analyzed by the Imagej software. GAPDH or Tublin was used to normalize the western spot values.

\section{Statistical analysis}

All statistical analyses were performed using SPSS 17.0 software (IBM Corp., Armonk, NY, USA). Wilcoxon rank sum test was used to 

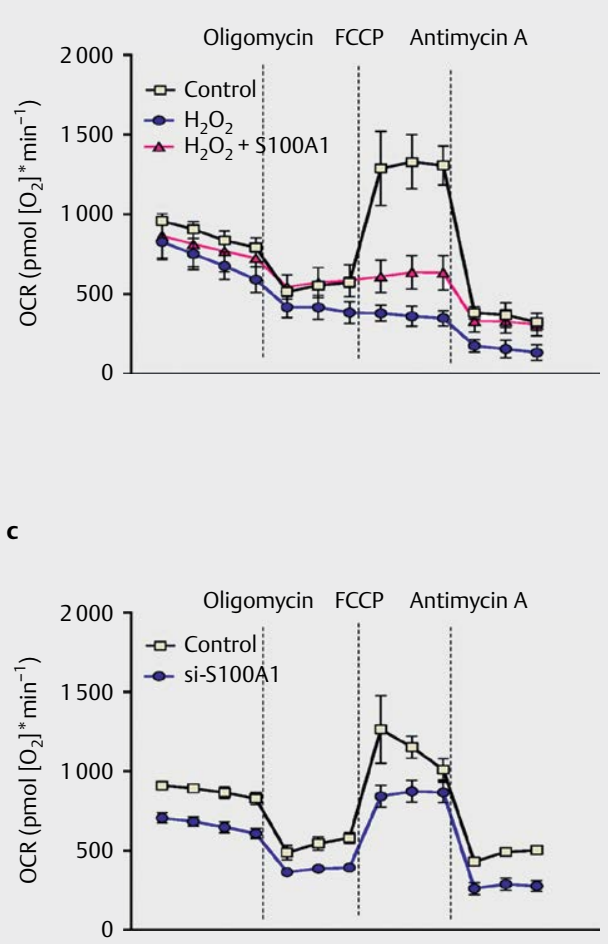

b

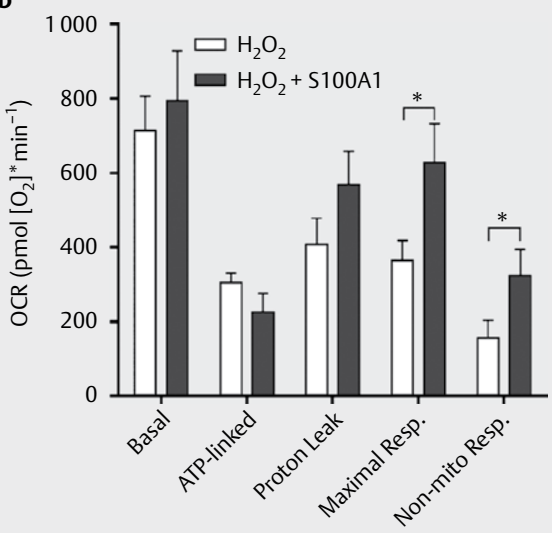

d

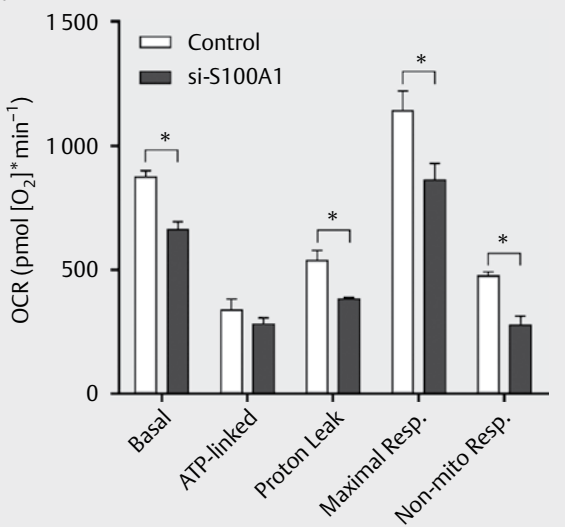

- Fig. 4 S100A1 increases maximal respiration, as assessed using the Seahorse system. (a) Oxygen consumption rate (OCR) of H9c2 (S100A1) and H9c2 (Vector) cells, with or without oxidative stress, was detected using the Seahorse system. (b) OCR was analyzed in several stages of respiration in H9c2 (S100A1) and H9c2 (Vector) cells with oxidative stress, including basal, ATP-linked, proton-leak, Maximal respiration, and non-mitochondrial respiration. (c) OCR of H9c2 (si-S100A1) and H9c2 (Nonsense) cells was detected using the Seahorse system. (d) OCR was analyzed in several stages of respiration in H9c2 (si-S100A1) and H9c2 (Nonsense) cells, including basal, ATP-linked, proton-leak, Maximal respiration, and non-mitochondrial respiration. An asterisk $\left({ }^{*}\right)$ indicates a significant change $(P<0.05, n=3)$

determine the significance of the differences between two groups. For the three or more groups of data, statistical analysis was performed using Kruskal-Wallis test. A two-sided value of $P<0.05$ was considered statistically significant.

\section{Results}

\section{Exhaustive exercise leads to decrease in mitochondrial antioxidants, increase ROS production, and myocardial injury in rats}

Cardiac cells require a continuous supply of energy for their function and thus contain a higher number of mitochondria to achieve their energy requirements [36]. Under conditions of EE, a highly oxidative metabolic environment predisposes the cardiac cells to free radical damage. Therefore, an EE model of rats was constructed to evaluate myocardial injury and oxidative stress under $\mathrm{EE}$. The results showed that the level of ROS in the plasma of the EE group was significantly higher than that of the control group ( $\vee$ Fig. 1a)
$(P<0.05)$. We also found that the level of SOD ( $\vee$ Fig. 1b) and GSHPX ( $\vee$ Fig. 1c) in the EE group was significantly lower than that in the control group $(P<0.05)$. Considering the correlation between oxidative stress and myocardial injury [37], we then examined the extent of the injury to cardiomyocytes. Exhaustive exercise led to a significant increase in plasma CK ( $\triangleright$ Fig. 1d), cTnT ( $\triangleright$ Fig. 1f), cTnl ( $\triangleright$ Fig. 1g) and CK-MB ( $\triangleright$ Fig. 1h) levels $(P<0.05)$. Hematoxylin and eosin staining showed that $E E$ resulted in myocardial damage, including disordered distribution of cardiomyocytes, blurred cell boundaries, myocardial fibers fracture, reticulated cytoplasm, and vacuolation ( Fig. 1e).

\section{Exhaustive exercise promotes myocardial mitochondria injury and downregulation of S100A1 in rats}

Considering that mitochondria are one of the important sites for ROS production [38], we detected the effect of exhausting exercise on the structure of mitochondria in vivo. As shown in > Fig. 2a, EE induced disordered and sparse myocardial fibers and swelling of 

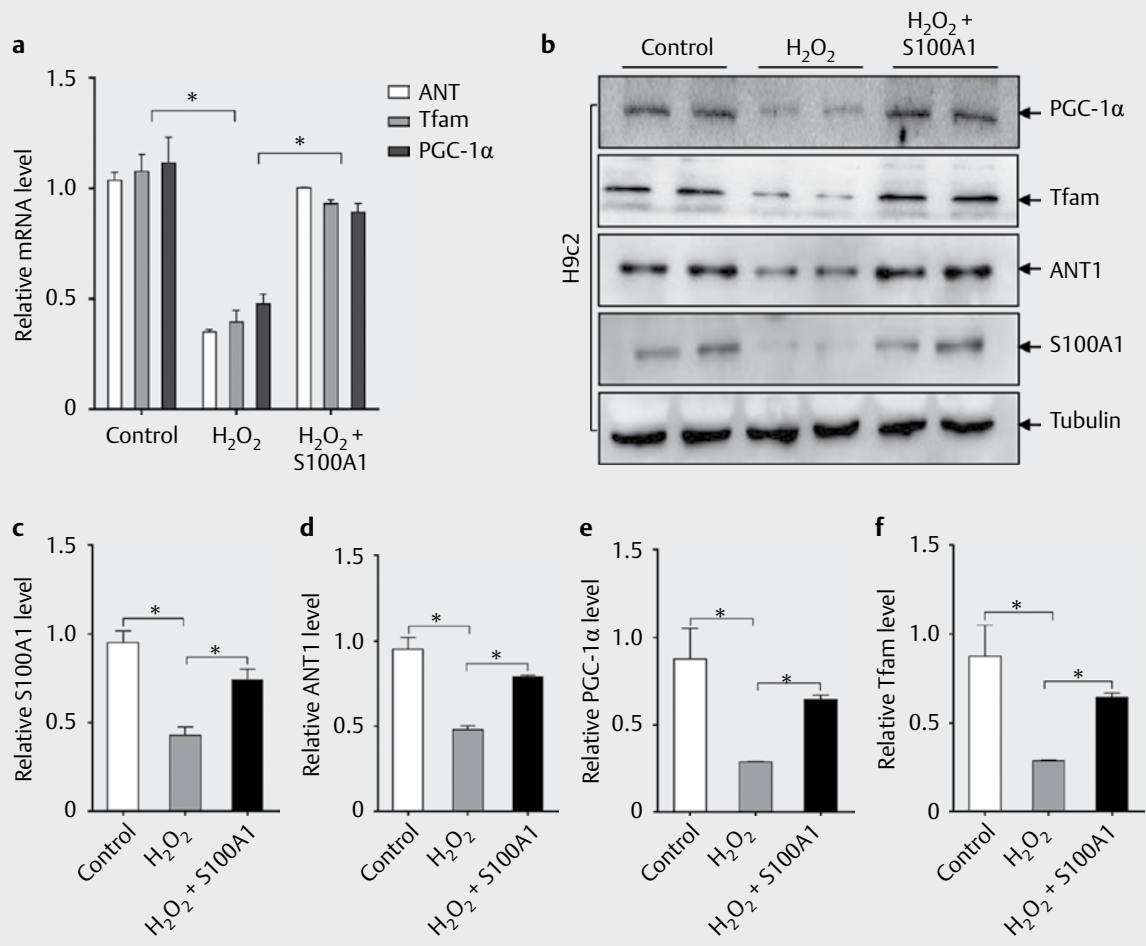

\begin{abstract}
- Fig. 5 Upregulation of S100A1 promotes the expression of Ant, Pgc1a, and Tfam in H9c2 cells. (a) Real-time PCR was carried out to determine the mRNA expression of Ant, Pgc1a, and Tfam using cDNA samples collected from $\mathrm{H} 9 \mathrm{c} 2$ cells ( $1 \mathrm{mM} \mathrm{H}_{2} \mathrm{O}_{2}$ for $24 \mathrm{~h}$ ), $\mathrm{H} 9 \mathrm{c} 2$ cells $\left(\mathrm{S} 100 \mathrm{~A} 1,1 \mathrm{mM} \mathrm{H} \mathrm{O}_{2}\right.$ for $24 \mathrm{~h}$ ), or $\mathrm{H} 9 \mathrm{c} 2$ cells (non-treated control). Values in the graphs represent the mean \pm standard deviation; an asterisk $\left({ }^{*}\right)$ indicates a significant change $(\mathrm{P}<0.05, \mathrm{n}=3)$. (b) Extracts of $\mathrm{H} 9 \mathrm{c} 2$ cells $\left(1 \mathrm{mM} \mathrm{H}_{2} \mathrm{O}_{2}\right.$ for $24 \mathrm{~h}$ ), $\mathrm{H} 9 \mathrm{c} 2$ cells $\left(\mathrm{S} 100 \mathrm{~A} 1,1 \mathrm{mM} \mathrm{H}_{2} \mathrm{O}_{2}\right.$ for $24 \mathrm{~h}$ ), or $\mathrm{H} 9 \mathrm{c} 2$ cells (non-treated control) were analyzed using western blotting. Tubulin was used as a protein loading control. (c-f) Quantitative analysis of ANT, PGC-1 $\alpha, S 100 A 1$, and Tfam levels in (B) using Image J. Values in the graphs represent the mean \pm standard deviation; an asterisk $\left({ }^{*}\right)$ indicates a significant change $(P<0.05, n=3)$.
\end{abstract}

mitochondria. S100A1 is a regulator of myocardial contractility. However, the relationship between S100A1 and mitochondrial function or oxidative stress is largely unknown. In the present study, S100a 1 expression was downregulated after EE in rats ( $\triangleright \mathbf{F i g} . \mathbf{2 b}$, - 2c \& d). These results indicate that $\mathrm{S100A1}$ might be related to mitochondrial dysfunction and oxidative stress after $\mathrm{EE}$.

\section{S100A1 protects H9c2 cells from oxidative stress in vitro}

To further explore the relationship between the decrease in S100A 1 expression and oxidative stress induced by EE, we used an S100a 1 overexpression vector to increase the level of $\mathrm{S} 100 \mathrm{~A} 1$ in cells under oxidative stress. The results showed that oxidative stress induced by $\mathrm{H}_{2} \mathrm{O}_{2}$ could decrease the cell survival rate in a dose-dependent ( $\vee$ Fig. 3a) and time-dependent ( $\vee$ Fig. 3b) manner. The mRNA level of S100A1 was significantly increased in H9c2 (S100A1) cells compared with H9c2 (Vector) cells under oxidative stress ( $\triangleright$ Fig. 3c). S100a1 overexpression significantly reduced the death rate ( $\triangleright$ Fig. 3d) of H9c2 cells induced by oxidative stress. Intracellular ROS levels of H9c2 (S100A1) also decreased compared with those in $\mathrm{H} 9 \mathrm{c} 2$ (Vector) cells under oxidative stress ( $\bullet$ Fig. 3e, f). In terms of intracellular antioxidant enzyme levels, S100a 1 overexpression led to a significant increase in SOD and GSH-PX level in oxidatively stressed $\mathrm{H} 9 \mathrm{c} 2$ cells ( $\bullet \mathbf{F i g} . \mathbf{3} \mathbf{g} \& \mathbf{h}$ ). The results illustrated that S100A 1 could inhibit oxidative stress in $\mathrm{H} 9 \mathrm{c} 2$ cells induced by $\mathrm{H}_{2} \mathrm{O}_{2}$, and reduce the injury and mortality of oxidative stress in $\mathrm{H} 9 \mathrm{c} 2$ cells. S100a1 overexpression significantly reduced ROS levels ( $\bullet$ Fig. $3 i$ ) and inhibited injury ( $\bullet$ Fig. 3j) in $\mathrm{H} 9 \mathrm{c} 2$ cells treated by $\mathrm{H}_{2} \mathrm{O}_{2}$.

\section{S100A1 increases maximal respiration by Seahorse}

The function of the mitochondrial respiration is coupled with the production of ROS in the form of superoxide anions or hydrogen peroxide [9]. Therefore, we tested the effect of S100A1 on mitochondrial respiratory function in oxidatively stressed cardiomyocytes. As shown in > Fig. 4a, $\mathrm{H}_{2} \mathrm{O}_{2}$ induced a significant decrease in mitochondrial respiratory function including, basal, ATP-linked, proton-leak, maximal respiration, and non-mitochondrial respiration. Upregulation of S100a1 showed a protective effect on mitochondrial respiration (maximal respiration and non-mitochondrial respiration) of $\mathrm{H} 9 \mathrm{c} 2$ cells under oxidative stress ( $\bullet \mathbf{F i g} . \mathbf{4 a} \& \mathbf{b}$ ). Based off our previous data that EE and $\mathrm{H}_{2} \mathrm{O}_{2}$ could lead to a decrease in S100a 1 expression and the oxidative stress response in cardiomyocytes, we decided to knock-down S100A 1 in cardiomyocytes. Thus, we introduced si-S100A1 into H9c2 cells to explore 


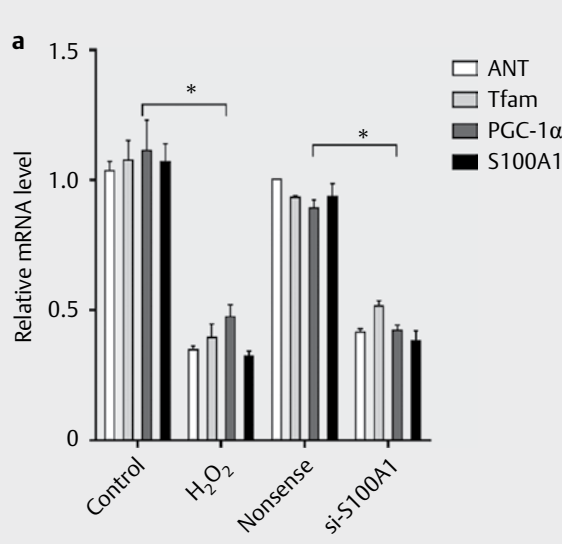

c

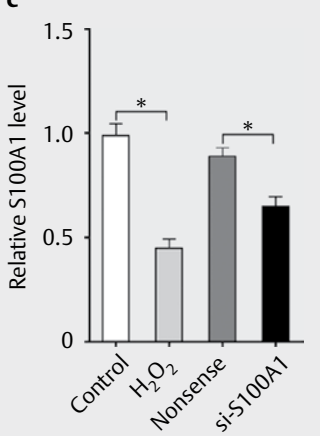

d

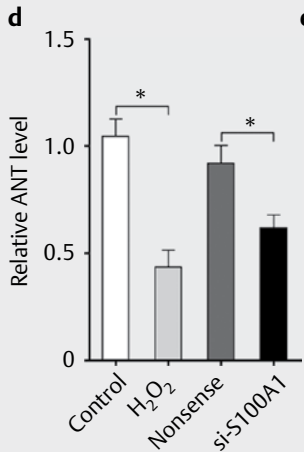

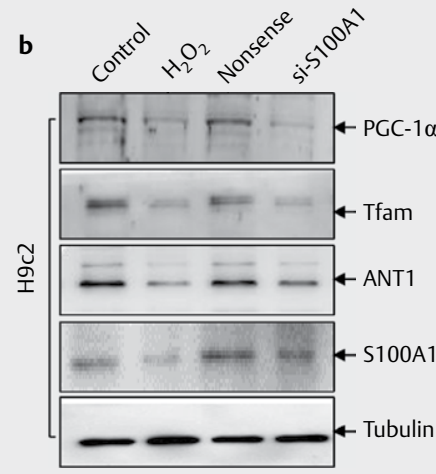

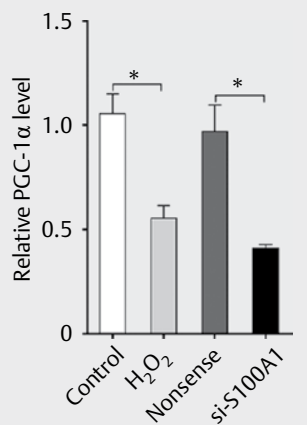

f

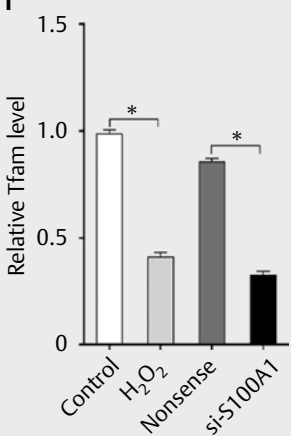

- Fig. 6 Knockdown of S100a1 inhibits the expression of Ant, Pgc1a, and Tfam in H9c2 cells. (a) Real-time PCR was carried out to determine the mRNA expression of Ant, Pgc1a, S100a1, and Tfam using cDNA samples collected from H9c2 cells ( $1 \mathrm{mM} \mathrm{H}_{2} \mathrm{O}_{2}$ for 24h), H9c2 cells (si-S100A1), H9c2 cells (Nonsense siRNA) or $\mathrm{H} 9 \mathrm{c} 2$ cells (non-treated control). Values in the graphs represent the mean \pm standard deviation; an asterisk $\left({ }^{*}\right)$ indicates a significant change $(P<0.05, n=3)$. (b) Extracts of $\mathrm{H} 9 \mathrm{c} 2$ cells $\left(1 \mathrm{mM} \mathrm{H}_{2} \mathrm{O}_{2}\right.$ for $\left.24 \mathrm{~h}\right)$, H9c2 cells (si-S100A1), H9c2 cells (Nonsense siRNA), or H9c2 cells (non-treated control) were analyzed using western blotting. Tubulin was used as a protein loading control. (c-f) Quantitative analysis of ANT, S100A1, PGC- $1 \alpha$ and Tfam levels in (B) using Image J. Values in the graphs represent the mean \pm standard deviation; an asterisk $\left({ }^{*}\right)$ indicates a significant change $(P<0.05, n=3)$.

the effect of S100A 1 on respiratory function. The results indicated that knockdown of S100a 1 led to a significant decrease in mitochondrial respiratory function, including basal, proton-leak, maximal respiration, and non-mitochondrial respiration ( $\mathbf{F i g . ~} \mathbf{4 c} \& \mathbf{d}$ ). These results suggested that $\mathrm{S100A} 1$ is an important molecule in the redox balance role of the mitochondria, which therefore allows for normal respiration to occur.

\section{S100A1 promotes the expression of ANT1, PGC-1 $\alpha$, and Tfam in $\mathrm{H} 9 \mathrm{c} 2$ cells}

For cells, the respiratory capacity of mitochondria is related to their quantity and function. Therefore, we detected the effect of S100A1 on expression of Ant1, Pgc1a, and Tfam, which are related to mitochondrial oxidative phosphorylation, transcription of energy metabolism genes, and mitochondrial genome replication, respectively. As shown in $>$ Fig. $\mathbf{5}, \mathrm{H}_{2} \mathrm{O}_{2}$ led to a significant reduction in Ant1, PGC-1 $\alpha$, and Tfam expression at the mRNA ( $\triangleright$ Fig. 5a) and protein ( $\triangleright$ Fig. $\mathbf{5 b}$ ) levels. Upregulation of S100a 1 in $\mathrm{H} 9 \mathrm{c} 2$ cells (treated with $\mathrm{H}_{2} \mathrm{O}_{2}$ ) promoted the expression of Ant1, Pgc1a, and Tfam ( $\vee$ Fig. 5b-f. Furthermore, the expression patterns of ANT1, PGC-1 $\alpha$, and Tfam in H9c2 cells (si-S100A1) were similar to those in $\mathrm{H} 9 \mathrm{c} 2$ cells (treated with $\mathrm{H}_{2} \mathrm{O}_{2}$ ). Specifically, downregulation of S100a 1 in H9c2 cells inhibited the mRNA expression ( $\bullet$ Fig. $\mathbf{6 a}$ ) and protein content ( $\vee$ Fig. $\mathbf{6 b}-\mathbf{f}$ ) of Ant1, Pgc1a, and Tfam. These results indicated that $\mathrm{S100A} 1$ is a key protein of mitochondrial oxidative phosphorylation, energy metabolism gene transcription, and mitochondrial genome replication via its regulation of ANT1, PGC- $1 \alpha$, and Tfam.

\section{Discussion}

Although free radicals were discovered in 1954, it was not until the 1970s that oxidative stress caused by muscle exercise was linked to body damage [39]. In recent years, death caused by EE and excessive fatigue has been gradually recognized, and the injury effects caused by EE have become a research focus. ROS produced in mitochondria participate in a variety of signaling and damaging pathways, regulating a variety of physiological and disease processes [40]. In the present study, our results showed that significantly increased ROS levels in the serum of exhausted rats were accompanied by myocardial tissue damage. Further testing found that plasma CK also increased significantly, while SOD and GSH-PX 


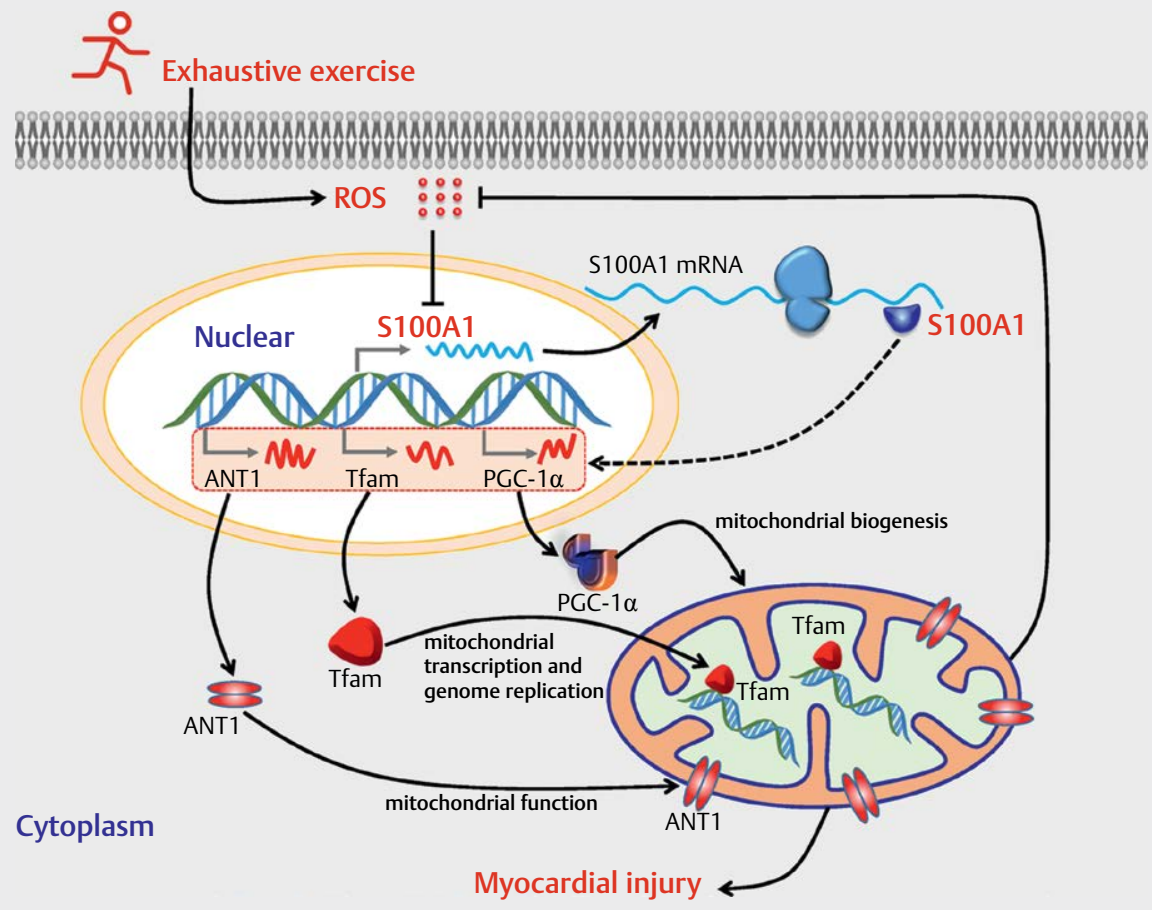

Pig. 7 Schematic diagram of the mechanism underlying S100A1's oxidative stress inhibition and mitochondria protection in H9c2 cells following exhaustive exercise.

levels in plasma decreased significantly ( $\triangleright$ Fig. 1 ). These results suggest that increased ROS after EE might be involved in the process of myocardial injury. However, the mechanism of myocardial injury during EE remains unclear.

Mitochondria are not only the source of energy supply during $\mathrm{EE}$, but also are one of the important sites of ROS production. In addition, mitochondria are an important target of ROS. Ultrastructural observation of the mitochondria in the myocardium of EE rats was performed using electron microscope. As shown in $>\mathbf{F i g . ~ 2 a , ~}$ EE induced disordered and sparse myocardial fibers and swelling of mitochondria. In vitro, we also confirmed that oxidative stress caused significant impairment of mitochondrial respiratory function ( $>$ Fig. 4a). In view of the important role of S100A1 in the protection of myocardial cell injury, and the relationship between $\mathrm{S} 100 \mathrm{~A} 1$ and exercise, we detected the expression of S100A 1 in the myocardium of EE rats. The results showed that EE led to a significant decrease in S100A1 levels as shown by immunohistochemistry ( $\triangleright$ Fig. 2d) and western blotting ( $\triangleright$ Fig. 2 b). Those results indicated that S100A1 downregulation in EE rats seemed to be involved in mitochondria and cardiomyocytes injury.

There are reports that $\mathrm{S100A} 1$ can regulate the inflammatory response and oxidative stress in $\mathrm{H} 9 \mathrm{C} 2$ cells via the TLR4/ROS/NF-KB pathway [41]. However, the regulatory effect of oxidative stress on S100A 1 and the feedback regulatory effect of S100A1 on ROS have not been explained clearly. To further explore the relationship between ROS and S100A1 expression, H9c2 cells were employed to model oxidative stress in vitro. We found that oxidative stress resulted in a significant decrease in cell survival ( $\triangleright$ Fig. 3 a \& b) and
S100a1 expression ( $\vee$ Fig. $\mathbf{3 c} \&$ Fig. $\mathbf{5 b}$ ), and that overexpression of $\$ 100 a 1$ reduced the level of oxidative stress ( $\vee$ Fig. $\mathbf{3 e} \& \mathbf{f}$ ) and increased the cell survival rate ( $\triangleright \mathbf{F i g}$. $3 \mathbf{d}$ ) in $\mathrm{H} 9 \mathrm{c} 2$ cells. These results suggested mutual regulation between ROS and S100A1. Mitochondrial function and ROS are also mutually regulated [42, 43]. Thus, we hypothesized that the relationship between ROS and S100A1 is related to mitochondrial function. The results of Seahorse system analysis showed that oxidative stress led to mitochondrial respiratory dysfunction including, basal, ATP-linked, protonleak, maximal respiration, and non-mitochondrial respiration, and S100a1 overexpression inhibited the effect of oxidative stress in H9c2 cells ( $\triangleright$ Fig. $4 a$ \& b). Inhibition of S100a 1 led to mitochondrial respiratory dysfunction in $\mathrm{H} 9 \mathrm{c} 2$ cells ( $\mathbf{F i g .} \mathbf{4 c} \& \mathbf{d}$ ). These results suggested that $\mathrm{S} 100 \mathrm{~A} 1$ is partly responsible for mitochondrial respiration dysfunction under oxidative stress in $\mathrm{H} 9 \mathrm{c} 2$ cells. Other mechanisms that can lead to myocardial injury during exhausting exercise are inhibiting autophagy, reducing mitochondrial function and increasing the level of oxidative stress $[44,45]$. There are limitations in the conclusion drawn only from oxidative stress cell model.

$\mathrm{S} 100 \mathrm{~A} 1$ is a regulator of $\mathrm{Ca}^{2+}$ in cardiomyocytes [46-48]. Specifically, enhancement of L-type calcium channel trans-sarcolemmal calcium influx by S100A via protein kinase $A$ has been reported [49]. Considering the relationship between calcium regulation and mitochondrial function in cells [50,51], it is not surprising that $\mathrm{Ca}^{2+}$ is one of the pathways by which $\mathrm{S} 100 \mathrm{~A} 1$ regulates mitochondrial function. To explore the non- $\mathrm{Ca}^{2+}$ mitochondrial regulatory pathway of S100A1, we detected the expression of ANT1, PGC-1 $\alpha$, and 
Tfam, which are related to mitochondrial oxidative phosphorylation [52], the transcription of energy metabolism genes [53], and mitochondrial genome replication [54] in oxidatively stressed H9c2 cells. The results showed that the expression levels of ANT1, PGC$1 \alpha$ and Tfam were significantly decreased in $\mathrm{H} 9 \mathrm{c} 2\left(\mathrm{H}_{2} \mathrm{O}_{2}\right)$ cells compared with those in $\mathrm{H} 9 \mathrm{c} 2$ cells. Overexpression of $\mathrm{S100a} 1$ reversed the decrease in ANT1, PGC- $1 \alpha$ and Tfam expression induced by $\mathrm{H}_{2} \mathrm{O}_{2}$ ( Fig. 5). Inhibition of S100a1 expression in $\mathrm{H} 9 \mathrm{c} 2$ cells achieved similar effects to those of oxidative stress ( $\triangleright$ Fig. $\mathbf{6}$ ). These results demonstrate the regulatory effect of S100A1 on ANT1, $\mathrm{PGC1}$, and Tfam on the transcriptomic level.

\section{Perspective}

As shown in the proposed schematic diagram in $\mathbf{F i g . ~ 7 , ~ o u r ~ r e - ~}$ sults found a novel effect of EE comprising S100A1 downregulation by ROS, which resulted in a decrease in the protein and mRNA levels of ANT1, PGC-1 $\alpha$, and Tfam. We also provided evidence that ROS act as a damage factor in cardiomyocytes under EE by promoting mitochondrial respiratory dysfunction. These findings suggested the function of S100A1 in EE-induced cardiomyocyte injury and provide novel insights into this key molecular mechanism.

\section{Declarations}

\section{Ethics approval and consent to participate}

This study was compliant with the Declaration of Helsinki Guidelines and the Ethical Standards in Sport and Exercise Science Research: 2020 Update [18].

\section{Availability of data and materials}

All data generated or analysed during this study are included in this published article.

\section{Funding}

This work was partially supported by the grants of the Natural Science Foundation of China [grant numbers 81373108, 31971106, 17-163-12-ZT-002-120-01, 18CXZ044].

\section{Conflicts of interest}

The authors declare no potential conflicts of interest

\section{References}

[1] Li Q, Tuo X, Li B et al. Semaglutide attenuates excessive exerciseinduced myocardial injury through inhibiting oxidative stress and inflammation in rats. Life Sci 2020; 250: 117531

[2] Fernandes T, Gomes-Gatto CV, Pereira NP et al. NO Signaling in the Cardiovascular System and Exercise. Adv Exp Med Biol 2017; 1000: 211-245
[3] Zimmer P, Bloch W. Physical exercise and epigenetic adaptations of the cardiovascular system. Herz 2015; 40: 353-360

[4] Chang Y, Yu T, Yang $\mathrm{H}$ et al. Exhaustive exercise-induced cardiac conduction system injury and changes of CTnT and Cx43. Int J Sports Med 2015; 36: 1-8

[5] Xu P, Wang Y, Sun W et al. Salidroside protects the cardiac function of exhausted rats by inducing Nrf2 expression. Cardiovasc J Afr 2020; 31 : 25-32

[6] Gajda R, Kowalik E, Rybka S et al. Evaluation of the heart function of swimmers subjected to exhaustive repetitive endurance efforts during a 500-km relay. Front Physiol 2019; 10: 296

[7] Ettema G, Oksnes M, Kveli E et al. The effect of exhaustive exercise on the choice of technique and physiological response in classical roller skiing. Eur J Appl Physiol 2018; 118: 2385-2392

[8] Kan NW, Huang WC, Lin WT et al. Hepatoprotective effects of Ixora parviflora extract against exhaustive exercise-induced oxidative stress in mice. Molecules 2013; 18: 10721-10732

[9] Angelova PR, Abramov AY. Role of mitochondrial ROS in the brain: from physiology to neurodegeneration. FEBS Lett 2018; 592: 692-702

[10] Mailloux RJ. An Update on mitochondrial reactive oxygen species production. Antioxidants (Basel) 2020; 9: 472

[11] Lin MT, Beal MF. Mitochondrial dysfunction and oxidative stress in neurodegenerative diseases. Nature 2006; 443: 787-795

[12] Kudryavtseva AV, Krasnov GS, Dmitriev AA et al. Mitochondrial dysfunction and oxidative stress in aging and cancer. Oncotarget 2016; 7: 44879-44905

[13] Turrens JF. Mitochondrial formation of reactive oxygen species. J Physiol 2003; 552: 335-344

[14] Grivennikova VG, Vinogradov AD. Mitochondrial production of reactive oxygen species. Biochemistry (Mosc) 2013; 78: 1490-1511

[15] Most P, Bernotat J, Ehlermann P et al. S100A1: A regulator of myocardial contractility. Proc Natl Acad Sci USA 2001; 98: 1388913894

[16] Rohde D, Schon C, Boerries M et al. S100A1 is released from ischemic cardiomyocytes and signals myocardial damage via Toll-like receptor 4. EMBO Mol Med 2014; 6: 778-794

[17] Fargnoli AS, Katz MG, Williams RD et al. Liquid jet delivery method featuring S100A1 gene therapy in the rodent model following acute myocardial infarction. Gene Ther 2016; 23: 151-157

[18] Harriss DJ, MacSween A, Atkinson G. Ethical standards in sport and exercise science research: 2020 update. Int J Sports Med 2019; 40: 813-817

[19] Bedford TG, Tipton CM, Wilson NC et al. Maximum oxygen consumption of rats and its changes with various experimental procedures. J Appl Physiol Respir Environ Exerc Physiol 1979; 47: 1278-1283

[20] Fu FH, Cen HW, Eston RG. The effects of cryotherapy on muscle damage in rats subjected to endurance training. Scand J Med Sci Sports 1997; 7: 358-362

[21] Yen CH, Tsao TH, Huang CU et al. Effects of sweet cassava polysaccharide extracts on endurance exercise in rats. I Int Soc Sports Nutr 2013; 10: 18

[22] Ba L, Gao J, Chen Y et al. Allicin attenuates pathological cardiac hypertrophy by inhibiting autophagy via activation of PI3K/Akt/mTOR and MAPK/ERK/mTOR signaling pathways. Phytomedicine 2019; 58: 152765

[23] Huang WQ, Wen JL, Lin RQ et al. Effects of mTOR/NF-kappaB signaling pathway and high thoracic epidural anesthesia on myocardial ischemia-reperfusion injury via autophagy in rats. J Cell Physiol 2018; 233: 6669-6678

[24] Ye N, Zhang N, Zhang Y et al. Cul4a as a New Interaction Protein of PARP1 Inhibits Oxidative Stress-Induced H9c2 Cell Apoptosis. Oxid Med Cell Longev 2019; 2019: 4273261 
[25] Zhang L, Liu Y, Li JY et al. Protective Effect of Rosamultin against H2O2-Induced Oxidative Stress and Apoptosis in H9c2 Cardiomyocytes. Oxid Med Cell Longev 2018; 2018: 8415610

[26] Yasuda J, Okada M, Yamawaki H. T3 peptide, an active fragment of tumstatin, inhibits $\mathrm{H} 2 \mathrm{O} 2$-induced apoptosis in $\mathrm{H} 9 \mathrm{c} 2$ cardiomyoblasts. Eur J Pharmacol 2017; 807: 64-70

[27] Santos AR, Lamas L, Ugrinowitsch C et al. Different resistance-training regimens evoked a similar increase in myostatin inhibitors expression. Int J Sports Med 2015; 36: 761-768

[28] Wang X, Feng Z, Li ] et al. High glucose induces autophagy of MC3T3-E1 cells via ROS-AKT-mTOR axis. Mol Cell Endocrinol 2016; 429: 62-72

[29] Zhang Z, Jiang F, Zeng L et al. PHACTR1 regulates oxidative stress and inflammation to coronary artery endothelial cells via interaction with NF-KB/p65. Atherosclerosis 2018; 278: 180-189

[30] Liu ], Ren Y, Hou Y et al. Dihydroartemisinin induces endothelial cell autophagy through suppression of the akt/mTOR pathway. J Cancer 2019; 10: 6057-6064

[31] Samadian Z, Azar JT, Moshari S et al. Moderate-intensity exercise training in sole and simultaneous forms with insulin ameliorates the experimental type 1 diabetes-induced intrinsic apoptosis in testicular tissue. Int J Sports Med 2019; 40: 909-920

[32] Hong F, Ze Y, Zhou Y et al. Nanoparticulate TiO2-mediated inhibition of the Wnt signaling pathway causes dendritic development disorder in cultured rat hippocampal neurons. J Biomed Mater Res A 2017; 105 : 2139-2149

[33] Liu XW, Lu MK, Zhong HT et al. Panax notoginseng saponins attenuate myocardial ischemia-reperfusion injury through the HIF-1alpha/BNIP3 pathway of autophagy. J Cardiovasc Pharmacol 2019; 73: 92-99

[34] Logan S, Pharaoh GA, Marlin MC et al. Insulin-like growth factor receptor signaling regulates working memory, mitochondrial metabolism, and amyloid-beta uptake in astrocytes. Mol Metab 2018; 9: $141-155$

[35] Shen C, Liu W, Zhang S et al. Downregulation of miR-541 induced by heat stress contributes to malignant transformation of human bronchial epithelial cells via HSP27. Environ Res 2019; 184: 108954

[36] Upadhyay S, Mantha AK, Dhiman M. Glycyrrhiza glabra (Licorice) root extract attenuates doxorubicin-induced cardiotoxicity via alleviating oxidative stress and stabilising the cardiac health in H9c2 cardiomyocytes. J Ethnopharmacol 2020; 258: 112690

[37] Law BA, Liao X, Moore KS et al. Lipotoxic very-long-chain ceramides cause mitochondrial dysfunction, oxidative stress, and cell death in cardiomyocytes. FASEB J 2018; 32: 1403-1416

[38] Ding ZM, Ahmad M], Meng F et al. Triclocarban exposure affects mouse oocyte in vitro maturation through inducing mitochondrial dysfunction and oxidative stress. Environ Pollut 2020; 262: 114271

[39] Powers SK, Radak Z, Ji LL. Exercise-induced oxidative stress: past, present and future. J Physiol 2016; 594: 5081-5092
[40] Wu W, Chang S, Wu Q et al. Mitochondrial ferritin protects the murine myocardium from acute exhaustive exercise injury. Cell Death Dis 2016; 7: e2475

[41] Yu J, Lu Y, Li Y et al. Role of S100A1 in hypoxia-induced inflammatory response in cardiomyocytes via TLR4/ROS/NF-kappaB pathway. J Pharm Pharmacol 2015; 67: 1240-1250

[42] Zhang X, Wang Y, Wei G et al. Stepwise dual targeting and dual responsive polymer micelles for mitochondrion therapy. J Control Release 2020; 322: 157-169

[43] Wei P, Yang F, Zheng $Q$ et al. The potential role of the nlrp3 inflammasome activation as a link between mitochondria ROS generation and neuroinflammation in postoperative cognitive dysfunction. Front Cell Neurosci 2019; 13: 73

[44] Liu HT, Pan SS. Late exercise preconditioning promotes autophagy against exhaustive exercise-induced myocardial injury through the activation of the AMPK-mTOR-ULK1 pathway. Biomed Res Int 2019; 2019: 5697380

[45] Zhang H, Liu M, Zhang Y et al. Trimetazidine attenuates exhaustive exercise-induced myocardial injury in rats via regulation of the $\mathrm{Nrf2/}$ NF-kappaB signaling pathway. Front Pharmacol 2019; 10: 175

[46] Most P, Remppis A, Pleger ST et al. Transgenic overexpression of the $\mathrm{Ca} 2+$-binding protein S100A1 in the heart leads to increased in vivo myocardial contractile performance. J Biol Chem 2003; 278 : 33809-33817

[47] Kettlewell S, Most P, Currie S et al. S100A1 increases the gain of excitation-contraction coupling in isolated rabbit ventricular cardiomyocytes. J Mol Cell Cardiol 2005; 39: 900-910

[48] Kiewitz R, Acklin C, Schafer BW et al. Ca2 + -dependent interaction of S100A1 with the sarcoplasmic reticulum Ca2 + -ATPase2a and phospholamban in the human heart. Biochem Biophys Res Commun 2003; 306: 550-557

[49] Reppel M, Sasse P, Piekorz R et al. S100A1 enhances the L-type Ca2 + current in embryonic mouse and neonatal rat ventricular cardiomyocytes. J Biol Chem 2005; 280: 36019-36028

[50] Boerries M, Most P, Gledhill JR et al. Ca2 + -dependent interaction of S100A1 with F1-ATPase leads to an increased ATP content in cardiomyocytes. Mol Cell Biol 2007; 27: 4365-4373

[51] Volkers M, Rohde D, Goodman C et al. S100A1: a regulator of striated muscle sarcoplasmic reticulum $\mathrm{Ca} 2+$ handling, sarcomeric, and mitochondrial function. J Biomed Biotechnol 2010; 2010: 178614

[52] Hoshino A, Wang W], Wada S et al. The ADP/ATP translocase drives mitophagy independent of nucleotide exchange. Nature 2019; 575: 375-379

[53] Dorn GW 2nd, Vega RB, Kelly DP. Mitochondrial biogenesis and dynamics in the developing and diseased heart. Genes Dev 2015; 29 : 1981-1991

[54] Murugesapillai D, McCauley M], Maher LJ 3rd et al. Single-molecule studies of high-mobility group B architectural DNA bending proteins. Biophys Rev 2017; 9: 17-40 